\title{
TOPOLOGICAL HOMOTHETIES ON COMPACT HAUSDORFF SPACES
}

\author{
LUDVIK JANOS
}

1. Introduction and terminology. Let $(X, \rho)$ be a metric space and $f: X \rightarrow X$ such that for some $c>0$ there exists a topologically equivalent metric $\rho^{*}$ such that $\rho^{*}(f(x), f(y))=c \rho^{*}(x, y)$ for all $x, y \in X$. In this case we call $f$ a topological c-homothety. If $X$ is compact metrizable then a simple purely topological condition characterizes this property. In fact in this case we have:

Theorem 1.1. Let $X$ be compact metrizable and $f: X \rightarrow X$ continuous. Then $f$ is a topological c-homothety for some $c \in(0,1)$ if and only if $f$ is one-to-one and $\cap f^{n}[X]=\{a\}$ for some $a \in X$. (The intersection of all iterated images of $X$ under $f$ is a singleton.) The proof of this theorem is in [3].

It is the purpose of this note to extend this result to nonmetrizable compact Hausdorff spaces, replacing the role of metrics by generating families of pseudometrics.

Let $X$ be a completely regular space. A family $\mathscr{D}=\left\{\rho_{\alpha} \mid \alpha \in \mathfrak{A}\right\}$ of pseudometrics $\rho_{\alpha}(x, y)$ on $X$ will be called a generating family on $X$ iff it generates the topology of $X$. (The system of sets $B(x, \epsilon, \alpha)$ $=\left\{y \mid \rho_{\alpha}(y, x)<\epsilon\right\}$ for all $\alpha \in \mathfrak{A}, x \in X$ and $\epsilon>0$ forms an open subbase for the topology of $X$.) The set of all such families will be denoted by $\$(X)$.

If $f: X \rightarrow X$ is such that for some $c>0 \rho_{\alpha}(f(x), f(y))=c \rho_{\alpha}(x, y)$ for all $\alpha \in \mathfrak{A}$ and all $x, y \in X, f$ is said to be a $c$-homothety with respect to $D=\left\{\rho_{\alpha} \mid \alpha \in \mathfrak{R}\right\} \in \mathscr{S}(X)$.

If $f: X \rightarrow X$ is such that for some $D \in(B)(X) f$ is a $c$-homothety with respect to $D$, we say $f$ is a topological c-homothety.

Let $X$ be an abstract set and $f: X \rightarrow X$. We will say $f$ is a squeezing mapping iff $\cap f^{n}[X]=\{a\}$ for some $a \in X$. This property will play a crucial role in our investigations. To illustrate this property we state the following theorem which will be used later:

Theorem 1.2 (A Converse of Banach's Contraction TheoREM). Let $X$ be compact metrizable and $f: X \rightarrow X$ a continuous and squeezing mapping. Then for any $c \in(0,1)$ there exists a metric $\rho(x, y)$

Presented to the Society, November 9, 1967; received by the editors May 10, 1968. 
on $X$, generating the topology of $X$, such that $f$ is a c-contraction with respect to $\rho$.

For the proof, see [4].

Remarking only that all spaces considered are Hausdorff we can state our main theorem:

Theorem. Let $X$ be compact and $f: X \rightarrow X$. Then $f$ is a topological $c$-homothety for some $c \in(0,1)$ iff $f$ is a homeomorphism into and a squeezing mapping.

As a corollary of this theorem and the theorem proved in [5] we will show that each topological $c$-homothety for $c \in(0,1)$ can be linearized in some linear topological space as a homothety in the usual sense. Finally we will also show that Theorem 1.2 can be generalized dropping the requirement of metrizability from its hypothesis.

2. Transformations of families of pseudometrics. Let $X$ be compact, $0<c \leqq 1, D \in\left(S(X), f: X \rightarrow X\right.$, and $D=\left\{\rho_{\alpha} \mid \alpha \in \mathfrak{U}\right\}$. For each $\alpha \in \mathfrak{A}$ we define

$$
\stackrel{*}{\rho_{\alpha}}(x, y)=\sup c^{n} \rho_{\alpha}\left(f^{n}(x), f^{n}(y)\right) .
$$

(The supremum is taken over the set of all nonnegative integers and $f^{0}(x)=x$.)

The resulting family $\left\{\rho_{\alpha}^{*} \mid \alpha \in \mathfrak{A}\right\}$ we denote by $\mathbb{D}(f, c)$.

Lemma 2.1. Let $X$ be compact, $f: X \rightarrow X$ continuous and $D \in B(X)$. Then (i) $D(f, c) \in(S(X)$ for each $c \in(0,1)$ and $c \rho(f(x), f(y)) \leqq \rho(x, y)$ for all $x, y \in X$ and $\rho \in D(f, c)$,

(ii) if $f$ is also squeezing, then $\mathscr{D}(f, 1) \in \mathscr{S}(X)$.

Proof. Let $f: X \rightarrow X$ be continuous,

$$
c \in(0,1) \text { and } D=\left\{\rho_{\alpha} \mid \alpha \in \mathfrak{N}\right\} \in \mathbb{S}(X) \text {. }
$$

First we have to show that for each $\alpha \in \mathfrak{Y}$ the function $\rho_{\alpha}^{*}(x, y)$ $=\sup c^{n} \rho_{\alpha}\left(f^{n}(x), f^{n}(y)\right)$ is a pseudometric. Since $\rho_{\alpha}$ is bounded there exists, for each $x, y \in X, n=n(x, y)$ such that $\rho_{\alpha}^{*}(x, y)=c^{n} \rho_{\alpha}\left(f^{n}(x), f^{n}(y)\right)$. Now let $x, y, z \in X$ be arbitrary elements. Let $m$ be such that

then we have

$$
\rho_{\alpha}^{*}(x, z)=c^{m} \rho_{\alpha}\left(f^{m}(x), f^{m}(z)\right) ;
$$

$$
\begin{gathered}
\rho_{\alpha}^{*}(x, y) \geqq c^{m} \rho_{\alpha}\left(f^{m}(x), f^{m}(y)\right), \\
\rho_{\alpha}^{*}(y, z) \geqq c^{m} \rho_{\alpha}\left(f^{m}(y), f^{m}(z)\right) .
\end{gathered}
$$


Applying the triangle inequality to $\rho_{\alpha}$ and the elements $f^{m}(x), f^{m}(y)$, $f^{m}(z)$ we get

$$
\begin{aligned}
\rho_{\alpha}^{*}(x, z) & =c^{m} \rho_{\alpha}\left(f^{m}(x), f^{m}(z)\right) \\
& \leqq c^{m} \rho_{\alpha}\left(f^{m}(x), f^{m}(y)\right)+c^{m} \rho_{\alpha}\left(f^{m}(y), f^{m}(z)\right) \\
& \leqq \rho_{\alpha}^{*}(x, y)+\rho_{\alpha}^{*}(y, z),
\end{aligned}
$$

which shows that $\rho_{\alpha}^{*}$ is a pseudometric.

From the definition it follows that

$$
\begin{aligned}
\rho_{\alpha}^{*}(f(x), f(y)) & =\sup c^{n} \rho_{\alpha}\left(f^{n+1}(x), f^{n+1}(y)\right) \\
& =c^{-1} \sup c^{n+1} \rho_{\alpha}\left(f^{n+1}(x), f^{n+1}(y)\right) \\
& \leqq c^{-1} \sup c^{m} \rho_{\alpha}\left(f^{m}(x), f^{m}(y)\right)=c^{-1} \rho_{\alpha}^{*}(x, y)
\end{aligned}
$$

which shows that

$$
c \rho_{\alpha}^{*}(f(x), f(y)) \leqq \rho^{*}(x, y) .
$$

Now we have to show that the original family $\left\{\rho_{\alpha}\right\}$ and the new family $\left\{\rho_{\alpha}^{*}\right\}$ generate the same topology. Because $\rho_{\alpha}(x, y) \leqq \rho_{\alpha}^{*}(x, y)$ for all $x, y \in X$ and all $\alpha \in \mathfrak{A}$, it is only necessary to show that for any converging net $\left\{x_{i}\right\} \rightarrow x(i \in D)$ the following proposition is true:

$$
\forall \alpha \in \mathfrak{A} \forall \epsilon>0 \exists i \in D\left[i \prec j \Rightarrow \rho_{\alpha}^{*}\left(x_{j}, x\right)<\epsilon\right]
$$

where $\prec$ denotes the partial order in $D$.

If this were not the case, then there would exist $\alpha \in \mathfrak{Y}$ and $\epsilon>0$ such that, for each $i \in D, \rho_{\alpha}^{*}\left(x_{j}, x\right) \geqq \epsilon$ for some $j \succ i$. This would mean that for each $i \in D$ there would exist a nonnegative integer $n=n(i)$ such that

$$
c^{n(i)} \rho_{\alpha}\left(f^{n(i)}\left(x_{j}\right), f^{n(i)}(x)\right) \geqq \epsilon
$$

for some $j \succ i$.

It follows from this inequality and the fact that $\rho_{\alpha}$ is bounded that the function $n(i)$ defined on $D$ must be bounded.

Let $n_{1}, n_{2}, \cdots, n_{k}$ be the values of this function; then considering the system of converging nets

$$
f^{n_{s}}\left(x_{i}\right) \rightarrow f^{n_{s}}(x) \quad \text { for } s=1,2, \cdots, k
$$

we get the desired contradiction of the above inequality, which proves (i).

To prove (ii) it is only necessary to realize that if $f$ is squeezing then the same contradiction can be obtained even if we put $c=1$. 
Evidently if

$$
\rho_{\alpha}\left(f^{n(i)}\left(x_{j}\right), f^{n(i)}(x)\right) \geqq \epsilon
$$

for some $j \succ i$, then the function $n(i)$ again must be bounded, otherwise there would exist sequences $j(k)$ and $n(k)$ such that

$$
f^{n(k)}(x) \rightarrow y \text { and } f^{n(k)}\left(x_{j(k)}\right) \rightarrow z \text { where } y \neq z
$$

which could contradict the fact that $\cap f^{n}(X)$ is a singleton.

Lемма 2.2. Let $Z$ be compact, $X \subset Z, D=\left\{\rho_{\alpha} \mid \alpha \in \mathfrak{A}\right\} \in(H(Z)$, $f: X \rightarrow X$ a homeomorphism of $X$ into itself such that for some $c \in(0,1)$, $\rho_{\alpha}(f(x),(f y)) \leqq c \rho_{\alpha}(x, y)$ for all $x, y \in X$ and all $\alpha \in \mathfrak{A}$, and finally let $g: Z \rightarrow Z$ be continuous and such that $g(x)=f^{-1}(x)$ for all $x \in f[X]$.

Then $f$ is a topological c-homothety on $X$.

For the proof we need only verify that $f$ is a $c$-homothety with respect to $D(g, c) \in(B)$.

3. Factorization of mappings. Let $X$ be completely regular and $\mathscr{D}=\left\{\rho_{\alpha} \mid \alpha \in \mathfrak{A}\right\} \in \mathfrak{S}(X)$. With each $\alpha \in \mathcal{S}$ there is associated an equivalence relation $R_{\alpha}$ on $X$ defined by $x R_{\alpha} y \Leftrightarrow \rho_{\alpha}(x, y)=0$. Denoting $X_{\alpha}=X / R_{\alpha}$ and by $p_{\alpha}(x)$ the class of all elements $R_{\alpha}$-equivalent to $x$, we have $p_{\alpha}: X \rightarrow X_{\alpha}$ for each $\alpha \in \mathfrak{A}$. Each $X_{\alpha}$ is canonically endowed with the metric $\rho_{\alpha}^{\prime}$ defined by

$$
\rho_{\alpha}^{\prime}\left(p_{\alpha}(x), p_{\alpha}(y)\right)=\rho_{\alpha}(x, y) .
$$

Forming the topological product $\prod_{\alpha} X_{\alpha}$ and defining $i: X \rightarrow \prod_{\alpha} X_{\alpha}$ by $i(x)=\left\{p_{\alpha}(x)\right\}$ (a typical element of $\prod_{\alpha} X_{\alpha}$ will be denoted by $\left.\left\{x_{\alpha}\right\}\right)$, we observe that $i$ is a continuous injection. If $X$ is compact then each $X_{\alpha}$ is compact and $i: X \rightarrow \prod_{\alpha} X_{\alpha}$ is a topological embedding.

If $f: X \rightarrow X$ is such that $x R_{\alpha} y \Rightarrow f(x) R_{\alpha} f(y)$ for all $\alpha \in \mathfrak{A}$ then $f$ induces a map $f_{\alpha}: X_{\alpha} \rightarrow X_{\alpha}$ on each $X_{\alpha}$ defined by

$$
f_{\alpha}\left(p_{\alpha}(x)\right)=p_{\alpha}(f(x)) \quad(\alpha \in \mathfrak{U}) .
$$

Defining $F: \prod_{\alpha} X_{\alpha} \rightarrow \prod_{\alpha} X_{\alpha}$ by $F\left\{x_{\alpha}\right\}=\left\{f_{\alpha}\left(x_{\alpha}\right)\right\}$ we see that the diagram

$$
\begin{gathered}
X \stackrel{i}{\rightarrow} \prod_{\alpha} X_{\alpha} \\
f \downarrow \\
X \stackrel{i}{\rightarrow} \prod_{\alpha} X_{\alpha}
\end{gathered}
$$

is commutative. 
It can be seen easily that if $f$ is nonexpansive with respect to D, i.e. $\rho_{\alpha}(f(x), f(y)) \leqq \rho_{\alpha}(x, y)$ for all $x, y \in X$ and all $\alpha \in \mathfrak{A}$, and squeezing, then $f$ preserves all relations $R_{\alpha}$ and the induced mappings $f_{\alpha}: X_{\alpha}$ $\rightarrow X_{\alpha}$ enjoy the same properties on $\left(X_{\alpha}, \rho_{\alpha}^{*}\right)$. This result enables us finally to prove our theorem.

REMARK 3.1. Let $\left\{\left(X_{\alpha}, \rho_{\alpha}\right) \mid \alpha \in \mathfrak{A}\right\}$ be a family of metric spaces and $Y=\prod_{\alpha} X_{\alpha}$ its topological product.

Defining the family $\left\{\rho_{\alpha}^{\prime \prime} \mid \alpha \in \mathfrak{X}\right\}$ on $Y$ by $\rho_{\alpha}^{\prime \prime}\left(\left\{x_{\beta}\right\},\left\{y_{\beta}\right\}\right)=\rho_{\alpha}\left(x_{\alpha}, y_{\alpha}\right)$, we observe easily that this belongs to $B(Y)$, and if we replace each metric $\rho_{\alpha}$ by a topologically equivalent metric $\rho_{1 \alpha}$ on $X_{\alpha}$ then the corresponding family $\left\{\rho_{1 \alpha}^{\prime \prime} \mid \alpha \in \mathfrak{A}\right\}$ again belongs to $\mathcal{S}(Y)$.

4. Proof of the theorem. If $X$ is a topological $c$-homothety for some $c \in(0,1)$ then it follows easily that $f$ is a homeomorphism and that the images $f^{n}[X]$ shrink to some point.

If conversely $f$ is a squeezing homeomorphism, then taking any $D \in S(X)$ and applying Lemma 2.1 , we see that $f$ is nonexpanding with respect to $D(f, 1) \in B(X)$. Therefore our factorization process described above is legitimate and each induced mapping $f_{\alpha}: X_{\alpha} \rightarrow X_{\alpha}$ is nonexpanding and squeezing on $X_{\alpha}$. Choosing $c \in(0,1)$, Theorem 1.2. yields the existence of a metric $\rho_{\alpha}$ on $X_{\alpha}$ such that $f_{\alpha}$ is a $c$-contraction on $\left(X_{\alpha}, \rho_{\alpha}\right)$ for each $\left(X_{\alpha}, \rho_{\alpha}\right)$ for each $\alpha \in \mathfrak{A}$. Each $\left(X_{\alpha}, \rho_{\alpha}\right)$ is compact and can be embedded into the Hilbert cube as a closed subset and, using the result of $\mathrm{R}$. H. Bing [1] which assures that a metric defined on a closed subset of a metrizable space can be extended to the whole space, $\rho_{\alpha}$ can be extended over this cube. Denoting by $\left(H_{\alpha}, \rho_{\alpha}\right)$ the metric space thus obtained and identifying $X$ with $i(X) \subset \prod_{\alpha} X_{\alpha} \subset \prod_{\alpha} H_{\alpha}=H$ we have the following situation:

$X$ is a closed subset of $H=\prod_{\alpha} H_{\alpha}$ and $f: X \rightarrow X$ a $c$-contraction with respect to the family $D=\left\{\rho_{\alpha} \mid \alpha \in \mathfrak{A}\right\} \in \mathscr{S}(H)$ (considered here as a family of pseudometrics on $H$ ). Now we observe that $f(X)$ is closed in $H$ and since $H$ is a Tychonov cube, Tietze's extension theorem yields the possibility of extending $f^{-1}: f(X) \rightarrow X$ over $H$. Denoting such a continuous extension of $f^{-1}$ by $g: H \rightarrow H$, we have all we need to apply Lemma 2.2. to show that $f$ is a topological $c$-homothety on $X$.

5. Linearization of topological homotheties. Following the ideas of J. de Groot laid down mainly in [2] we will present still another characterization of topological homotheties, using a linear topological space as a space into which the given space will be embedded and where the given mapping will act as a homothety in the usual sense.

Definition 5.1. Let $X$ be completely regular and $f: X \rightarrow X$. We say that $f$ can be linearized in $L$ as a $c$-homothety iff there exists a linear 
topological space $L$, a number $c>0$ and a topological embedding $i: X \rightarrow L$ in such a way that $i(f(x))=c i(x)$ for all $x \in X$.

Lemma 5.1. Let $X$ be compact metrizable and $f: X \rightarrow X$. Then the following two statements are equivalent:

(1) $f$ is a topological $c$-homothety for some $c \in(0,1)$,

(2) $f$ can be linearized in a separable Hilbert space as a c-homothety for some $c \in(0,1)$.

The proof of this lemma is in [5], and we will show that this result can be generalized to the nonmetrizable case.

Theorem 5.1. Let $X$ be compact and $f: X \rightarrow X$. Then the following two statements are equivalent:

(1) $f$ is a topological c-homothety on $X$ for some $c \in(0,1)$,

(2) $f$ can be linearized as a c-homothety in some linear topological space for some $c \in(0,1)$.

Proof. Suppose (2) is true, then obviously $f$ is continuous and one-to-one and therefore a homeomorphism, and since $c^{n} i(X)$ shrinks to zero in $L$ as $n \rightarrow \infty, f$ is also squeezing, thus a topological c-homothety.

Suppose now (1) is true, then taking $D=\left\{\rho_{\alpha} \mid \alpha \in \mathfrak{U}\right\} \in \mathbb{S}(X)$ with respect to which $f$ is a $c$-homothety, we observe that the corresponding mappings $f_{\alpha}: X_{\alpha} \rightarrow X_{\alpha}$ are now $c$-homotheties on the metric spaces $\left(X_{\alpha}, \rho_{\alpha}\right)$. Using Lemma 5.1, we can consider each $X_{\alpha}$ to be embedded in a separable Hilbert space $\mathfrak{S}_{\alpha}$ in such a way that the mapping $f_{\alpha}: X_{\alpha} \rightarrow \mathfrak{S}_{\alpha}$ acts as multiplying by $c$ in $\mathfrak{S}_{\alpha}$. Defining $L=\prod_{\alpha} \mathfrak{W}_{\alpha}$ we have established (2). Q.E.D.

Combining this result with our main theorem, our final statement reads as follows:

Let $X$ be compact and $f: X \rightarrow X$. Then the following statements are equivalent:

(1) $f$ is a topological c-homothety for some $c \in(0,1)$.

(2) $f$ is a squeezing homeomorphism.

(3) $f$ can be linearized in some linear topological space $L$ as a chomothety for some $c \in(0,1)$.

REMARK. It follows from the proof of Theorem 5.1 that statement (3) can be modified to require that $L$ be locally convex.

Now we apply the results of $\S \S 2$ and 3 to generalize Theorem 1.2.

6. A converse of the generalized Banach's contraction theorem. Definition 6.1. Let $X$ be completely regular and $D \in(S)(X)$. If $f: X \rightarrow X$ is such that for some $c \in(0,1)$ we have $\rho(f(x), f(y)) \leqq c \rho(x, y)$ for all $\rho \in D$ and all $x, y \in X$ we say that $f$ is a contraction with respect to $\$$. 
If for $c \in(0,1)$ there exists $D \in B(X)$ such that $f$ is a $c$-contraction with respect to $D$ we say that $f$ is a topological c-contraction on $X$.

Proposition 6.1. If $f: X \rightarrow X$ is such that for some $c \in(0,1)$ there exists $\mathbb{D} \in(S)(X)$ such that:

(i) $f$ is a c-contraction with respect to $D$,

(ii) $X$ is complete with respect to D. (If a sequence is $\rho$-Cauchy for all $\rho \in D$ then it converges.)

Then $f$ has a unique fixed point $a \in X$ and $f^{n}(x) \rightarrow a$ for all $x \in X$. If we further assume that each $\rho \in D$ is bounded then $f$ is squeezing.

The proof of this proposition follows immediately from the fact that for any $x \in X$ the sequence $\left\{f^{n}(x)\right\}$ is $\rho$-Cauchy for all $\rho \in D$, and if $\rho$ is bounded then $\rho$-diameters of $f^{n}[X]$ tend to zero.

If $X$ is compact we have as a corollary the following theorem:

Theorem 6.1. Let $X$ be compact and $f: X \rightarrow X$ continuous. Then $f$ is a topological c-contraction for any $c \in(0,1)$ if and only if $f$ is squeezing.

Proof. If $f$ is a topological $c$-contraction for some $c \in(0,1)$ then Proposition 6.1. yields that $f$ is squeezing. If $f$ is squeezing and continuous then Lemma 2.1. shows the possibility of choosing a $D \in S(X)$ with respect to which $f$ is nonexpansive. Then the factorization process is legitimate with this $\mathscr{D} \in \mathscr{S}(X)$ and if we denote $\mathscr{D}=\left\{\rho_{\alpha} \mid \alpha \in \mathfrak{A}\right\}$ we get that for each $\alpha \in \mathfrak{A}$ the induced mapping $f_{\alpha}: X_{\alpha} \rightarrow X_{\alpha}$ is continuous and squeezing. Thus choosing $c \in(0,1)$ arbitrarily, Theorem 1.2. yields an existence of metrics $\rho_{1 \alpha}$ on each $X_{\alpha}$ such that $f_{\alpha}$ is a $c$-contraction on $\left(X_{\alpha}, \rho_{1 \alpha}\right)$. Identifying $X$ with $i(X)$ it follows from Remark 3.1. that there exists a family $D^{*} \in(S)(Y)\left(Y=\prod_{\alpha} X_{\alpha}\right)$ such that $F$ is a $c$-contraction with respect to it, which yields our theorem.

The author would like to express his gratitude to Professor Michael Edelstein for his valuable advice and improvements.

\section{REFERENCES}

1. R. H. Bing, Extending a metric, Duke Math. J. 14 (1947), 511-519.

2. J. de Groot, Linearization of a homeomorphism, Math. Ann. 144 (1961), 80-92.

3. L. Janos, Topological homotheties on compact metrizable spaces, Amer. J. Math. 90 (1968), 877-880.

4. - A converse of the Banach's contraction theorem, Proc. Amer. Math. Soc. 18 (1967), 287-289.

5. - Linearization of a contractive homeomorphism, Amer. J. Math. 90 (1968), 881-884.

6. M. Edelstein, $A$ proof of a theorem of L. Janos, Proc. Amer. Math. Soc. 20 (1969), 509-510.

UNIVERSITY OF FLORIDA 